\title{
Borovik-Poizat rank and stability
}

\author{
Jeffrey Burdges* \\ Department of Mathematics, Rutgers University \\ Hill Center, Piscataway, New Jersey 08854, U.S.A \\ e-mail: burdges@math.rutgers .edu \\ Gregory Cherlin ${ }^{\dagger}$ \\ Department of Mathematics, Rutgers University \\ Hill Center, Piscataway, New Jersey 08854, U.S.A \\ e-mail: cherlin@math.rutgers.edu
}

November 12, 2018

\section{Introduction}

Borovik proposed an axiomatic treatment of Morley rank in groups, later modified by Poizat, who showed that in the context of groups the resulting notion of rank provides a characterization of groups of finite Morley rank [Poi87]. (This result makes use of ideas of Lascar, which it encapsulates in a neat way.) These axioms form the basis of the algebraic treatment of groups of finite Morley rank undertaken in [BN94].

There are, however, ranked structures, i.e. structures on which a BorovikPoizat rank function is defined, which are not $\aleph_{0}$-stable [BN94, p. 376]. In

\footnotetext{
*Supported by NSF Graduate Research Fellowship

†Supported by NSF Grant DMS 9803417
} 
[Poi87, p. 9] Poizat raised the issue of the relationship between this notion of rank and stability theory in the following terms: “... un groupe de Borovik est une structure stable, alors qu'un univers rangé n'a aucune raison de l'être ..." (emphasis added). Nonetheless, we will prove the following:

Theorem 1.1 A ranked structure is superstable.

An example of a non- $\aleph_{0}$-stable structure with Borovik-Poizat rank 2 is given in [BN94, p. 376]. Furthermore, it appears that this example can be modified in a straightforward way to give $\aleph_{0}$-stable structures of Borovik-Poizat rank 2 in which the Morley rank is any countable ordinal (which would refute a claim of [BN94, p. 373, proof of C.4]). We have not checked the details. This does not leave much room for strenghthenings of our theorem. On the other hand, the proof of Theorem 1.1 does give a finite bound for the heights of certain trees of definable sets related to unsuperstability, as we will see in $\$ 5$

Since Shelah gave combinatorial criteria both for instability as well as for unsuperstability in a stable context, to prove the theorem we need only show that these criteria are incompatible with the Borovik-Poizat rank axioms. Now the rank axioms apply only to one structure, while Shelah's criteria take their simplest form in a saturated model. There are two ways to bridge this gap. Our first proof worked directly within the model in which the rank function is defined, paying attention in the process to the uniformity of various first order definitions. In the proof we give here, we first extract the first order content of the rank axioms, then work with them directly in a saturated model.

We will present the original rank axioms together with a few basic consequences in $\S 2$. Their first order content is analyzed in $\S 3$; we call the rank notions that result $B P_{0}$-ranks. In $\S 4$ we prove stability, and superstability is proved in $\S 5$. The proof of superstability does not depend on the full strength of the axioms, so we will develop the basic facts about rank in a more general context adequate for these applications. 
For the stability and rank issues that concern us here, we may always assume that the language of the structure involved is countable, and we take advantage of this in $\S 3$.

\section{The Borovik-Poizat Axioms and variations}

Let $\mathcal{M}$ be a structure. Let $\mathcal{D}$ be the collection of parametrically definable subsets of $\mathcal{M}^{\text {eq }}$, i.e. the sets and relations interpretable in $\mathcal{M}$. We say that $\mathcal{M}$ is a ranked structure [BN94, p. 57] if there is a rank function $\mathrm{rk}:(\mathcal{D}-\{\emptyset\}) \rightarrow \mathbb{N}$ which satisfies the following axioms for all $A, B \in \mathcal{D}$. Such a rank will be called a $B P$-rank.

Axiom 1 (Monotonicity of $\operatorname{rank}$ ) $\operatorname{rk}(A) \geq n+1$ iff there are infinitely many pairwise disjoint, nonempty, definable subsets of $A$, each of rank at least $n$.

Axiom 2 (Definability of rank) If $f$ is a definable function from $A$ to $B$ then for each integer $n$ the set $\left\{b \in B: \operatorname{rk}\left(f^{-1}(b)\right)=n\right\}$ is definable.

Axiom 3 (Additivity of rank) If $f$ is a definable function from $A$ onto $B$, and for all $b \in B$ we have $\operatorname{rk}\left(f^{-1}(b)\right)=n$, then $\operatorname{rk}(A)=\operatorname{rk}(B)+n$.

Axiom 4 (Elimination of infinite quantifiers) For any definable function $f$ from $A$ into $B$ there is an integer $m$ such that for any $b \in B$ the preimage $f^{-1}(b)$ is infinite whenever it contains at least $m$ elements.

We adopt the convention that $\operatorname{rk}(\emptyset)=-\infty$.

These axioms are unnecessarily strong for our purposes. We prefer to work with the following weaker form of Axiom 1, and to omit additivity of rank entirely:

Axiom 1.1 (Weak monotonicity) $\operatorname{rk}(A \cup B)=\max (\operatorname{rk}(A), \operatorname{rk}(B))$

Axiom 1.2 (Finite degree) If there are infinitely many pairwise disjoint, nonempty, definable subsets of $A$, each of rank at least $n$, then $\operatorname{rk}(A) \geq n+1$ 
Let "Axiom 1'" mean Axioms 1.1 and 1.2. A rank satisfying Axioms 1', 2, and 4 will be called a $B P^{\prime}$-rank.

Axiom 1.1 is easily derived from full monotonicity as in BN94, Lemma 4.10 p. 59], and of course Axiom 1.2 is simply part of Axiom 1, so a BP-rank is a $B P^{\prime}$-rank.

The following special case of Axiom 1.1 is often used without comment:

Fact 2.1 [BN94, Lemma 4.9 p. 59] In a ranked structure $\mathcal{M}^{\mathrm{eq}}$, if $A \subseteq B$ are two definable sets in $\mathcal{M}^{\mathrm{eq}}$, then $\operatorname{rk}(A) \leq \operatorname{rk}(B)$.

Two of our axioms are more conveniently phrased in terms of uniformly definable families of sets. A family of sets $\left\{S_{i}: i \in I\right\}$ is uniformly definable over the structure $\mathcal{M}$ if there is a single formula $\phi(x, y)$ defined in $\mathcal{M}^{\mathrm{eq}}$ and a choice of parameters $b_{i}(i \in I)$ such that $S_{i}=\phi\left[\mathcal{M}^{\mathrm{eq}}, b_{i}\right]$ for all $i$. With the formula $\phi$ fixed, we write $S_{b}$ for $\phi\left[\mathcal{M}^{\text {eq }}, b\right]$. Note that the formula $\phi(x, y)$ involves no parameters, as their places are filled by the variables $y$.

Proposition 2.2 Let $\mathcal{M}$ be structure with a rank function satisfying Axioms 2 and 4 , let $D$ be a definable subset of $\mathcal{M}^{\mathrm{eq}}$, and let $\left\{S_{b}\right\}_{b \in D}$ be a uniformly definable family of sets over $\mathcal{M}$. Then:

1. (Definability of rank) For each integer $n,\left\{b \in D: \operatorname{rk}\left(S_{b}\right)=n\right\}$ is definable. [BN94, Lemma 4.23 p. 66]

2. (Elimination of infinite quantifiers) There is a bound $m$, depending only on the formula $\phi$, on the size of the finite members of the family $\left\{S_{b}\right\}$.

A property of rank which is sometimes taken for granted is invariance under definable bijections. We do not know if this holds for $B P^{\prime}$-ranks in general, and we will not assume it.

Definition 2.3 Let $\mathcal{M}$ be a structure with a rank satisfying Axiom 1.1, and let $A, B$ be two definable sets in $\mathcal{M}^{\mathrm{eq}}$. We write $A \equiv B$ if the rank of their 
symmetric difference is less than the rank of their union. This may also be stated as follows: they have the same rank $n$, and the rank of their symmetric difference is less than $n$.

Observe that by Axiom 1.1 this relation is an equivalence relation.

Remark 2.4 In Axiom 2, or equivalently in Proposition 2.2, part (1), we refer to definability with parameters from the model $\mathcal{M}$. If the language of $\mathcal{M}$ is countable, then we may take the set of parameters involved to be countable as well.

\section{Canonical extensions of definable ranks}

Whenever one has a definable rank function on a structure $\mathcal{M}$, it has a canonical extension to any elementary extension of $\mathcal{M}$; details are given below. We study the canonical extensions of $B P$-ranks or $B P^{\prime}$-ranks in the present section, giving axioms which are satisfied by these ranks in general, and which exactly characterize these canonical extensions in the case in which the language is countable.

The focus of interest is Axiom 1.2, which could be phrased as follows: every set has a degree (analogous to the Morley degree). We will review the theory of the degree below, and show how to replace Axiom 1.2 by a degree approximation property which holds in canonical extensions. First we deal with the issue of the "canonical extension."

Definition 3.1 Let $\mathcal{M}$ be a structure equipped with a definable rank notion, that is a function from definable subsets of $\mathcal{M}^{\mathrm{eq}}$ to $\mathbb{N}$ such that for any uniformly definable family $\left\{S_{b}\right\}$ over $\mathcal{M}$, the set

$$
\left\{b: \operatorname{rk}\left(S_{b}\right)=n\right\}
$$

is definable (Axiom 2), and suppose that the rank has the following monotonicity property: if $A \subseteq B$ then $\operatorname{rk}(A) \leq \operatorname{rk}(B)$ (a consequence of Axiom 1.1). This 
implies that the rank is bounded on any uniformly definable family of sets. Let $\mathcal{N}$ be an elementary extension of $\mathcal{M}$. The canonical extension of rk to $\mathcal{N}$ (again denote "rk") is defined as follows. Every parametrically definable subset B of $\mathcal{M}^{\text {eq }}$ has a canonical extension $B^{*}$ to $\mathcal{N}^{\text {eq }}$. For $S \subseteq \mathcal{N}^{\text {eq }}$ definable, let $\operatorname{rk}(S)=n$ iff there is a uniformly definable family $\left\{S_{b}: b \in B\right\}$ indexed by a parametrically definable subset of $\mathcal{M}$ such that $\operatorname{rk}\left(S_{b}\right)=n$ for $b \in B$, and $S=S_{b^{*}}$ for some $b^{*} \in B^{*}$. (We have to check that this produces a well-defined rank function.)

Lemma 3.2 With the hypotheses and notation of the preceding definition, every definable subset of $\mathcal{N}^{\mathrm{eq}}$ is assigned a well-defined rank by the canonical extension of the rank function from $\mathcal{M}$ to $\mathcal{N}$.

Proof:

Let $S$ be definable in $\mathcal{N}^{\text {eq }}$. Then we can find a uniformly definable family $\left(S_{b}: b \in B\right)$ with $B$ 0-definable, such that $S=S_{b^{*}}$ for some $b^{*} \in B$. Furthermore each set $S_{b}$ is contained in a single sort of $\mathcal{N}^{\mathrm{eq}}$, and by our (very weak) monotonicity hypothesis, $\operatorname{rk}\left(S_{b}\right)$ is bounded for $b \in B$. Hence by definability of ranks, $B^{\mathcal{M}}$ can be partitioned into a finite number of $\mathcal{M}$-definable sets $B_{i}$ such that $\operatorname{rk}\left(S_{b}\right)=i$ on $B_{i}$. Then $b^{*} \in B_{i}^{\mathcal{N}}$ for some $i$, and hence we get $\operatorname{rk}\left(S_{b}\right)=i$. Thus every definable set is assigned at least one rank.

We must also verify that no conflicts arise. Suppose therefore that $S$ lies in the extension to $\mathcal{N}$ of the uniformly definable families $\left\{S_{b}: b \in B\right\}$ and $\left\{T_{c}: c \in C\right\}$, and that rk is constant on both families. Since $\mathcal{N}$ is an elementary extension of $\mathcal{M}$, it follows that in $\mathcal{M}$ there are some $b, c$ such that $S_{b}=T_{c}$. Hence the ranks are equal.

It is easy to see that any one of Axioms 1.1,2,3,4 will be preserved by the passage to a canonical extension if it holds in the original model. Our main interest at the moment will be in Axiom 1.2 (and later, in the rest of Axiom 1).

Definition 3.3 If $\mathcal{M}$ is a structure with a rank function satisfying Axiom 1.1, and $A$ is a definable set in $\mathcal{M}^{\mathrm{eq}}$, of rank $n$, then we say that $A$ has degree $d$ 
if $A$ can be decomposed into $d$ disjoint definable pieces of rank $n$, but no more. We say that $A$ has a degree (or, for emphasis, a finite degree) if it has degree $d$ for some $d$. When $A$ has a degree, we use $\operatorname{deg} A$ to denote the degree of $A$.

The theory of degree for the case of $B P$-rank is dealt with in BN94, Lemma $4.12,4.13]$. In our context this reads as follows:

Fact 3.4 Let $\mathcal{M}$ be a structure with a rank function rk satisfying Axiom 1.1. Then

1. If $A$ is a definable set in $\mathcal{M}^{\mathrm{eq}}$ which has rank $n$ and degree $d$, then $A$ may be partitioned into $d$ definable pieces $A_{i}(1 \leq i \leq d)$ of rank $n$ and degree 1 , and for any definable subset $B$ of $A$ of rank $n$ and degree 1, we have $B \equiv A_{i}$ for a unique $i$. In particular, the partition is unique modulo sets of lower rank in the following sense: if $A$ is also decomposed as the union of d definable subsets $A_{i}^{\prime}$ of rank $n$ and degree 1 , then after a permutation of the indices, we will have $A_{i} \equiv A_{i}^{\prime}$.

2. If $A, B$ are disjoint definable sets of equal rank which have degrees, then $\operatorname{deg}(A \cup B)=\operatorname{deg}(A)+\operatorname{deg}(B)$.

3. If the rank function satisfies Axiom $1^{\prime}$ then every set has a degree.

As a substitute for the existence of degree, we consider the following, which can be stated loosely in the form: "sets of finite degree are dense." As usual, we consider a rank function defined over a structure $\mathcal{M}$; and we also fix a set of parameters $C \subseteq \mathcal{M}$.

(Degree Approximation Property over $C$ ) If $A$ is a nonempty $C$ definable set, and $\left\{S_{a}: a \in A\right\}$ is a uniformly definable family (in particular, $S_{a}$ is $a$-definable for each $a$ ), then there is an element $a_{0} \in A$ for which $\operatorname{deg}\left(S_{a_{0}}\right)$ is finite. 
The case $C=\emptyset$ is reasonably strong but we will need this relativized version. The case $C=M$ (the universe of $\mathcal{M}$ ) is pointless, as we are then assuming that every definable set has a degree, since each definable set, taken by itself, constitutes a $C$-definable family in this case.

Definition 3.5 $A B P_{0}^{\prime}$-rank on a structure $\mathcal{M}$, relative to a set of parameters $C$, is a function $f: \mathcal{D} \backslash\{\emptyset\} \rightarrow \mathbb{N}$ satisfying:

Axiom 1.1 Weak Monotonicity: $\operatorname{rk}(A \cup B)=\max (\operatorname{rk}(A), \operatorname{rk}(B))$

Axiom $1.2^{\prime}$ The Degree $C$-approximation property

Axiom 2 Definability of rank, with parameters in $C$ (i.e. Proposition 2.2, with parameters in $C$ ).

Axiom 4 Elimination of Infinite Quantifiers

Lemma 3.6 Let $\mathcal{M}$ be a structure equipped with a $B P^{\prime}$-rank rk, for which the rank function is definable with parameters in $C \subseteq \mathcal{M}$. Let $\mathcal{N}$ be an elementary extension of $\mathcal{M}$. Then the canonical extension of $\mathrm{rk}$ to $\mathcal{N}$ is a $B P_{0}^{\prime}$-rank relative to the same set of parameters. Furthermore, the canonical extension satisfies the additivity axiom if and only if the original rank function does.

Proof:

In the context of a definable rank, the additivity property is clearly first order, so the final claim is immediate.

We should however check the $C$-approximation property for degree. As every nonempty $C$-definable set $B$ has a point in $\mathcal{M}$, and every $\mathcal{M}$-definable set $S$ has a degree, we just have to check that the degree of $S$ is unaltered by canonical extension. This reduces to the case in which $S$ has degree 1 . So suppose that in $\mathcal{N}$ we have a definable subset $T$ of $S^{*}$ so that both $T$ and $S^{*} \backslash T$ have rank $n=\operatorname{rk}(S)$. Using definability of rank we can pull this down to a set $T_{0}$ defined in $\mathcal{M}$ with $T_{0}$ and $S \backslash T_{0}$ of rank $n$, a contradiction. 
One may refine this slightly: the canonical extension of a $B P_{0}^{\prime}$-rank is again a $B P_{0}^{\prime}$-rank, by essentially the same argument. When the language is countable, our axioms actually characterize the canonical extensions of $B P^{\prime}$-ranks, as will be seen in the proof of the next result.

Theorem 3.7 Let $T$ be a complete theory in a countable language. Then the following conditions are equivalent:

(A) T has a model with a $B P^{\prime}$-rank.

(B) T has a model with a BP $P_{0}^{\prime}$-rank.

(C) $T$ has a countable extension by constants $T_{C}$, such that every model of $T_{C}$ carries a $B P_{0}^{\prime}$-rank, for which the degree approximation property, and definability of rank, hold relative to the empty set.

The equivalence of conditions $(B)$ and $(C)$ is clear, but worth noting, and worth using: by passing to $T_{C}$ we can work over the empty set, and lighten the notation. For the proof that $(C)$ implies $(A)$ we rely on the following.

Lemma 3.8 Let $T$ be a complete theory in a countable language, and suppose that $T$ has a model $\mathcal{M}$ with a $B P_{0}^{\prime}$-rank relative to $\emptyset$. Then $T$ has a prime model.

Proof:

We must show that $T$ is atomic, i.e. that every $\emptyset$-definable nonempty set $X$ contains an $\emptyset$-definable atom. We may assume $X$ has minimal rank since ranks are finite. By degree approximation $X$ has a degree, since the family $\{X\}$ is already definable over the empty set. We may suppose that $\operatorname{deg}(X)$ is minimized as well; we then claim that $X$ is an atom over $\emptyset$.

By weak monotonicity any $\emptyset$-definable nonempty subset $Y$ of $X$ will have rank no larger than $\operatorname{rk}(X)$, and hence equal to $\operatorname{rk}(X)$ by the minimization; accordingly $Y$ will have finite degree no greater than $\operatorname{deg}(X)$, and hence equal 
to $\operatorname{deg}(X)$; the same cannot apply simultaneously to $X \backslash Y$, so $X \backslash Y$ must be empty, and $Y=X: X$ is an atom over $\emptyset$.

Proof of Theorem 3.7

We assume $\mathcal{N}$ is a structure on which we have a $B P_{0}^{\prime}$-rank rk which has the degree approximation property and definability of rank relative to the empty set. By Lemma 3.8 the theory of $\mathcal{N}$ has a prime model $\mathcal{M}$, which we take to be an elementary substructure of $\mathcal{N}$. We claim that on $\mathcal{M}$, the rank function gives a $B P^{\prime}$-rank. Only Axiom 1.2 presents any issues: we claim that every $\mathcal{M}$-definable set $S$ has a degree.

Let $S$ be $a$-definable with $a \in M$ (an $n$-tuple for some $n)$. Let $A$ be the locus of $a$ over the empty set; as the type of $a$ is principal, $A$ is a 0 -definable set. By the degree approximation property, $A$ contains a point $a^{\prime}$ for which the corresponding set $S_{a^{\prime}}$ has a degree $d$. As $\operatorname{tp}(a)=\operatorname{tp}\left(a^{\prime}\right)$ and rank is $\emptyset$-definable, it follows easily that $\operatorname{deg}\left(S_{a}\right)=d$ as well.

We will show that the existence of $B P_{0}^{\prime}$-rank implies superstability. This is of course equivalent to the statement that a $B P^{\prime}$-rank gives superstability, since the problem localizes to countable languages. As this is our main application, we have emphasized $B P^{\prime}$-ranks and $B P_{0}^{\prime}$-ranks. However we can treat $B P$-ranks similarly.

Definition 3.9 Let $\mathrm{rk}: \mathcal{D} \backslash\{\emptyset\} \rightarrow \mathbb{N}$ be a rank function over a structure $\mathcal{M}$, and $C \subseteq \mathcal{M}$ a set of parameters. We say that rk has the splitting property if every set of rank $n>0$ contains a definable subset of rank $n-1$, and we say that rk has the splitting approximation property over $C$ if for every uniformly definable family of infinite sets $\left\{S_{b}: b \in B\right\}$ indexed by a nonempty $C$-definable set $B$, there is an element $b \in B$ such that $S_{b}$ contains a definable subset $S^{\prime}$ with $\operatorname{rk}\left(S^{\prime}\right)=\operatorname{rk}\left(S_{b}\right)-1$.

Observe that a $B P^{\prime}$-rank is a $B P$-rank if and only if it has the splitting property and additivity. We define a $B P_{0}$-rank relative to a set of parameters 
$C$ analogously, as a rank satisfying Axiom 1.1, degree and splitting approximation, and Axioms $2-4$, where the degree approximation property, the splitting approximation property, and the definability of rank all hold over $C$.

Theorem 3.10 Let $T$ be a complete theory in a countable language. Then the following conditions are equivalent:

(A) T has a model with a BP-rank.

(B) T has a model with a BP $P_{0}$-rank.

(C) $T$ has a countable extension by constants $T_{C}$, such that every model of $T_{C}$ carries a $B P_{0}$-rank, for which the degree approximation property, definability of rank, and the splitting approximation property all hold relative to the empty set.

Proof:

As before we need only prove $(C \Rightarrow A)$, and this reduces to the claim that a $B P_{0}$-rank on the prime model is a $B P$-rank, with the only property not yet verified being the splitting property. Again, this reduces to the claim that if $\operatorname{tp}(a)=\operatorname{tp}\left(a^{\prime}\right)$ and we have a uniformly definable family for which $S_{a^{\prime}}$ contains a definable subset $S^{\prime}$ with $\operatorname{rk}\left(S^{\prime}\right)=\operatorname{rk}\left(S_{a^{\prime}}\right)-1$, then the same applies to $S_{a}$. This is clear by definability of rank.

\section{Generic indistinguishability and Stability}

Before taking up stability as such, we analyze the structure of definable binary relations in general. For this it will be convenient to introduce a quantifier " $\forall^{*} x$," read "for generic $x$," as follows.

Definition 4.1 Assume $\mathcal{M}$ carries a definable rank function satisfying Axiom 1.1. Let $X$ be a definable set. " $\left(\forall^{*} x \in X\right) \psi(x)$ " means: " $\psi$ holds generically 
on $X$, " i.e. $\operatorname{rk}(X-\psi[X])<\operatorname{rk}(X)$. By the definability of rank, if $X=X_{a}$ and $\psi=\psi(x, b)$ both vary over uniformly definable families, the set $\{(a, b)$ : $\left.\forall^{*} x \in X_{a} \psi(x, b)\right\}$ is definable. In other words, first order logic is closed under the quantifier $\forall^{*}$.

By Axiom 1.1, if $\psi_{1}$ and $\psi_{2}$ hold generically on $X$, then so does $\psi_{1} \& \psi_{2}$. On the other hand, the property: "For all $\psi, \psi$ holds generically or $\neg \psi$ holds generically" is equivalent to the condition that the degree of $X$ is equal to 1 . Note also that the relation $A \equiv B$ defined above can be expressed as follows: $\left(\forall^{*} x \in A \cup B\right)[x \in A \Longleftrightarrow x \in B]$.

Definition 4.2 Let $\mathcal{M}$ be a structure with a definable rank function satisfying Axiom 1.1. Let $R$ be a definable relation on a definable set $S$. We will say that $x_{1}, x_{2} \in S$ are generically indistinguishable for $R$ on $S$, and we write $x_{1} \sim x_{2}$, if $\left(\forall^{*} x \in S\right)\left(R\left(x_{1}, x\right) \Longleftrightarrow R\left(x_{2}, x\right)\right)$.

Observe that this is an equivalence relation, and is definable from whatever parameters are needed to define $R$ and $S$, together with those used to define rank.

Proposition 4.3 Let $\mathcal{M}$ be a structure with a $B P_{0}^{\prime}$-rank. Let $S$ be a definable set in $\mathcal{M}^{\mathrm{eq}}$ and $R$ a definable binary relation on $S$. Let $\sim$ be the relation of generic indistinguishability for $R$ on $S$. Then $S / \sim$ has finitely many classes.

Proof:

First, put $S$ and $R$ into a uniformly definable family $\left\{\left(S_{a}, R_{a}\right): a \in A\right\}$ with $A$ defined over the empty set. Let $\sim_{a}$ be the relation of generic indistinguishability relative to $R_{a}$ on $S_{a}$. Note that $\sim_{a}$ is definable from the parameter $a$ together with parameters needed to define certain ranks. Then $\left\{S_{a} / \sim_{a}\right\}$ is a uniformly definable collection of sets. Hence there is a uniform bound $m$ on the sizes of its finite members. Consider $I=\left\{a:\left|S_{a} / \sim_{a}\right|>m\right\}$, the set of 
indices for which the quotient is infinite. Our claim is that $I$ is empty. If rank is $C$-definable, then $I$ is also $C$-definable.

Suppose $I$ is nonempty. Then there is some $a \in I$ such that $d=\operatorname{deg}\left(S_{a}\right)<$ $\infty$. We will show that $S_{a} / \sim_{a}$ has only finitely many classes to obtain a contradiction.

$S_{a}$ may be partitioned into $d$ definable pieces $S_{a, i}$ of degree 1 . For $x_{1} \in S_{a}$, the set $\left\{x \in S_{a}: R_{a}\left(x_{1}, x\right)\right\}$ coincides with a union of some of the $S_{a, i}$, modulo sets of lower rank. In other words, there is a set $S^{\prime}$, a union of finitely many of the $S_{a, i}$, for which:

$$
\left(\forall^{*} x \in S_{a}\right)\left[R_{a}\left(x_{1}, x\right) \Longleftrightarrow x \in S^{\prime}\right]
$$

As there are at most $2^{d}$ possibilities for $S^{\prime}$, the relation $\sim_{a}$ has at most $2^{d}$ classes. As this is finite, we have the desired contradiction.

Theorem 4.4 Let $\mathcal{M}$ be a structure with a $B P_{0}^{\prime}$-rank. Then $\operatorname{Th}(\mathcal{M})$ is stable.

Proof:

We may replace $\mathcal{M}$ by any elementarily equivalent model. So if the theory of $\mathcal{M}$ is unstable, we may suppose that there is a definable relation $R$ on a definable subset $X$ of $\mathcal{M}^{\text {eq }}$ such that $R$ linearly orders some infinite subset of $X$, not necessarily definable. We may also assume that $\mathcal{M}$ is $\omega_{1}$-saturated. Let $S$ be a definable set which contains an infinite subset $L$ which is linearly ordered by $R$, and has minimal rank.

Now we consider the relation $\sim$ of generic indistinguishability for $R$ on $S$. Since $S / \sim$ is finite, one of the equivalence classes for $\sim$ on $S$ meets $L$ in an infinite set. So without loss of generality $S$ consists of a single $\sim$-class. As $\mathcal{M}$ is $\omega_{1}$-saturated we may suppose $L$ has the order type of the rationals.

Consider elements $a, b \in L$ with $a<b$. The set $S^{\prime}=\{x \in S:[R(a, x) \Longleftrightarrow$ $\neg R(b, x)]\}$ contains the interval $(a, b)$ of $L$, hence by the minimality of $\operatorname{rk}(S)$ we 
find

$$
\operatorname{rk}\left(S^{\prime}\right)=\operatorname{rk}(S)
$$

But this violates the generic indistinguishability of $a$ and $b$.

\section{Superstability}

Theorem 5.1 Let $\mathcal{M}$ be a structure with a $B P_{0}^{\prime}$-rank. Then $\operatorname{Th}(\mathcal{M})$ is superstable.

Proof:

In this proof we will be less cavalier about the distinction between elements and $k$-tuples, as this will permit a slight refinement of the result (see the remark following the proof).

Suppose $\mathcal{M}$ is an unsuperstable structure with a $B P_{0}^{\prime}$-rank. As $\operatorname{Th}(\mathcal{M})$ is stable by the previous theorem, we can apply a combinatorial criterion due to Shelah, involving an infinitely branching tree of infinite height whose levels consist of pairwise disjoint uniformly definable sets. This goes as follows.

In the first place we have $D^{1}(x=x, L, \infty)=\infty$ She90, Theorem II 3.14 p. 53]. Therefore, by [She90, Lemma VII 3.5(5) p. 423], there are formulas $\phi_{k} \in L$ for $0 \leq k<\omega$ and in some model $\mathcal{M}^{\prime}$ of $T$ there are parameters $a_{v}$ for $v$ a node of the tree $\omega^{<\omega}$, such that:

(i) If $v \leq w$ are two nodes in the tree, then $\phi_{|w|}\left[\mathcal{M}^{\prime}, a_{w}\right] \subseteq \phi_{|v|}\left[\mathcal{M}^{\prime}, a_{v}\right]$ and $\phi_{|w|}\left[\mathcal{M}^{\prime}, a_{w}\right] \neq \emptyset$

(ii) For any two distinct nodes $v, w$ at the same level $k$ of the tree,

$$
\phi_{k}\left[\mathcal{M}^{\prime}, a_{v}\right] \cap \phi_{k}\left[\mathcal{M}^{\prime}, a_{w}\right]=\emptyset .
$$

Note that the only condition imposed on the (single) root formula $\phi_{0}\left[\mathcal{M}^{\prime}, a_{\emptyset}\right]$ is that $\phi_{0}\left[\mathcal{M}^{\prime}, a_{\emptyset}\right]$ should contain all the sets $\phi_{1}\left[\mathcal{M}^{\prime}, a_{<i>}\right]$. We may assume that $r_{0}=\operatorname{rk}\left(\phi_{0}\left[\mathcal{M}^{\prime}, a_{\emptyset}\right]\right)$ is minimal among all such trees. 
For parameters $b$ of the same sort as $a_{\emptyset}$, consider the parameters giving possible first level nodes of full rank

$$
N_{b}=\left\{c: \phi_{1}\left[\mathcal{M}^{\prime}, c\right] \subseteq \phi_{0}\left[\mathcal{M}^{\prime}, b\right] \text { and } \operatorname{rk}\left(\phi_{1}\left[\mathcal{M}^{\prime}, c\right]\right)=r_{0}\right\}
$$

Let $c_{1} \sim_{b} c_{2}$ be the relation defined on $N_{b}$ by $\phi_{1}\left[\mathcal{M}^{\prime}, c_{1}\right] \equiv \phi_{1}\left[\mathcal{M}^{\prime}, c_{2}\right] . N_{b}$ and $\sim_{b}$ are uniformly definable (by definability of rank) and $\sim_{b}$ is an equivalence relation.

Claim 5.1.1 $N_{a} / \sim_{a}$ has finitely many classes

Proof:

First, $\left\{N_{x} / \sim_{x}\right\}$ is a uniformly definable collection of sets, so there is a uniform bound $m$ on the sizes of its finite members. Let $C=\left\{x:\left|N_{x} / \sim_{x}\right|>m\right\}$ be the set of indices of infinite ones. If $C \neq \emptyset$ then there is a $b \in C$ such that $d=\operatorname{deg}\left(\phi_{0}\left[\mathcal{M}^{\prime}, b\right]\right)<\infty$. Now, $N_{b} / \sim_{b}$ has at most $2^{d}$ classes, contradiction.

This indicates that there are only finitely many first level nodes of full rank and there exists an index $i_{0}$ such that $\operatorname{rk}\left(\phi_{1}\left[\mathcal{M}^{\prime}, a_{<i_{0}>}\right]\right)<r_{0}$. We find a contradiction to the choice of $r_{0}$ by taking $\phi_{j}^{\prime}=\phi_{j+1}$ for $j \geq 0$ and $a_{v}^{\prime}=a_{<i_{0}, v>}$.

Remark 5.2 We can prove a stronger result: The height of any such tree of nonempty definable subsets of $\mathcal{M}^{\prime}$, pairwise disjoint at each level, with infinite branching, cannot exceed $r=\operatorname{rk}\left(\phi_{0}\left[\mathcal{M}^{\prime}, a_{\emptyset}\right]\right) \leq \operatorname{rk}\left(\mathcal{M}^{\prime}\right)$. This goes by induction on $r$ by following the line of the previous argument.

\section{References}

[BN94] Alexandre Borovik and Ali Nesin. Groups of Finite Morley Rank. The Clarendon Press Oxford University Press, New York, 1994. Oxford Science Publications. 
[Poi87] Bruno Poizat. Groupes stables. Nur al-Mantiq wal-Ma'rifah, Villeurbanne, 1987.

[She90] S. Shelah. Classification Theory and the Number of Nonisomorphic Models. North-Holland Publishing Co., Amsterdam, second edition, 1990. 